\title{
Un modèle d'extraction des collocations en langue de spécialité
}

\section{François Maniez}

\section{OpenEdition}

\section{Journals}

Édition électronique

URL : http://journals.openedition.org/asp/1597

DOI : 10.4000/asp. 1597

ISBN : 978-2-8218-0388-6

ISSN : 2108-6354

Éditeur

Groupe d'étude et de recherche en anglais de spécialité

Édition imprimée

Date de publication : 1 décembre 2002

Pagination : 35-47

ISSN : 1246-8185

Référence électronique

François Maniez, "Un modèle d'extraction des collocations en langue de spécialité », ASp [En ligne], 35-36 | 2002, mis en ligne le 07 août 2010, consulté le 19 avril 2019. URL : http://

journals.openedition.org/asp/1597; DOI : 10.4000/asp.1597

Ce document a été généré automatiquement le 19 avril 2019

Tous droits réservés 


\title{
Un modèle d'extraction des collocations en langue de spécialité
}

\author{
François Maniez
}

\section{Introduction}

1 L'une des principales caractéristiques des langues de spécialité est leur haute densité terminologique. Cette prédominance quantitative de la terminologie fait de l'extraction terminologique un champ d'investigation privilégié en traitement automatique des langues. Mais l'un de ses effets secondaires est également de rendre plus délicate l'extraction phraséologique et l'étude des collocations. Les expressions de patrons syntaxiques identiques (par exemple celles du type $<$ Adjectif - Nom> en anglais) ${ }^{1}$ peuvent être repérées automatiquement grâce à un étiquetage morpho-syntaxique, mais un étiquetage tenant compte de traits sémantiques est nécessaire pour séparer les termes des simples collocations.

2 L'étude des collocations en langue de spécialité est une activité qui a été considérablement facilitée par la mise au point de logiciels d'extraction et de concordance. Parmi les travaux les plus récents, on peut citer ceux de G. Williams dans le domaine de la biologie végétale et ceux de M.-C. L'Homme dans le domaine de l'informatique. Williams (1998) recherche les cooccurrences significatives entre deux lexèmes, non seulement afin d'extraire des binômes ou des expressions polylexicales mais aussi pour déterminer leur "rôle thématique, facteur de cohésion textuelle», ce qui l'amène à utiliser la notion de "réseaux de collocation ", qu'il représente par ailleurs sous forme graphique. Il utilise pour le repérage des collocations en corpus l'indice du MI score de Church et Hanks. Les réseaux ainsi définis décrivent en fait une combinatoire lexicale et grammaticale des termes du domaine. Ainsi, à partir du repérage d'un terme ( ptDNA), on peut découvrir un « encadrement collocationnel » (the ptDNA of), qui peut luimême être intégré à des modèles de taille supérieure (in the ptDNA of <plant_name, genes/ regions in the ptDNA of <plant_name>). À partir de notions centrales du domaine comme 
celle de germination et de résistance, Williams regroupe les exemples faisant intervenir les formes nominales, verbales et adjectivales pour arriver à la définition de ses réseaux. M.-C. L'Homme concentre principalement son étude sur les collocations à base verbale, en particulier dans le domaine de l'informatique (1998). Elle effectue la description des verbes spécialisés dans une optique de traitement automatique et la situe à différents niveaux (syntaxique, sémantique et combinatoire). À partir d'emplois du vocabulaire informatique tels que «tourner sur ", qu'elle formalise par la formule Act1(logiciel) tourne (1) sur Act2(ordinateur), elle établit un modèle descriptif qui s'inspire de la lexicologie explicative et combinatoire de Mel'cuk (1984). L'étude des corpus du domaine informatique lui a permis de décrire la structure actancielle de plus de 200 acceptions verbales spécialisées dans le domaine de l'informatique.

3 L'intérêt que manifeste L'Homme pour la description des structures verbales se justifie pleinement dans le cadre du TALN ${ }^{2}$, car si les progrès de l'extraction terminologique sont rapides, les problèmes que pose le traitement automatique du groupe verbal, notamment dans le cas de la traduction assistée par ordinateur, rendent cette formalisation indispensable. La description des collocations verbales en langue de spécialité comporte cependant un certain nombre d'écueils. Le premier est la faible fréquence des formes verbales en langue de spécialité relativement aux groupes nominaux, qui implique l'utilisation de corpus de très grande taille si l'on souhaite atteindre la significativité statistique. Le second est la nécessité du recours à un corpus arboré3 ${ }^{3}$ pour la détection de l'ensemble des structures faisant intervenir des syntagmes prépositionnels, la longueur des groupes nominaux objets n'assurant pas une proximité suffisante entre ces syntagmes prépositionnels et les verbes dont ils dépendent pour qu'une simple recherche de cooccurrents donne des résultats fiables.

\section{Repérage des collocations en langue de spécialité : le problème de la polysémie}

Quelques exemples tirés de la langue médicale nous serviront à illustrer la difficulté de la détection des collocations en langue de spécialité. La première difficulté réside dans l'absence de séparation nette entre vocabulaire spécialisé et vocabulaire général, phénomène dû à la polysémie de nombreux mots d'usage courant. Nous avons ainsi examiné les 134 occurrences du mot lit dans un corpus composé des polycopiés des Facultés de médecine de trois universités françaises ${ }^{4}$. L'examen manuel de ces formes fait apparaître deux types d'emploi distincts : ceux dans lesquels le lit désigne l'objet matériel (surélévation de la tête du lit, repos au lit, (geste) réalisé/ réalisable au lit du patient, pouvant être utilisé au lit du malade, confiné au lit, garder le lit) et ceux dans lesquels il désigne une partie de l'anatomie et précède dans la plupart des cas un adjectif relationnel à valeur localisante (lit vasculaire / artériel / vésiculaire / capillaire). Qu'il s'agisse de la détection des collocations ou de l'extraction terminologique, la prise en compte des statistiques de probabilité de cooccurrence nécessiterait idéalement un traitement séparé des exemples de chacun de ces emplois, de la même manière que les dictionnaires traitent les homographes dans des entrées distinctes, en distinguant lit1 de lit2. On décompte également neuf emplois de l'expression "faire le lit de" dans son sens étiologique (exemple: Les infarctus osseux qui accompagnent le processus infectieux semblent faire le lit des localisations septiques). La fréquence relativement élevée dans notre corpus de cette expression de la langue générale nous indique qu'elle constitue sans doute un élément de 
la phraséologie médicale qui vaut la peine d'être répertorié. Mais l'arsenal actuel du TALN ne permet pas le repérage automatique de combinaisons lexicales de mots d'une aussi haute fréquence d'emploi. Même dans un corpus lemmatisé qui regrouperait les emplois des cinq formes du verbe faire présentes dans le corpus, l'indice de probabilité de cooccurrence de faire et de lit resterait extrêmement bas du fait de leur haute fréquence d'emploi, et ce quel que soit le mode de calcul utilisé.

\section{Repérage des collocations disjointes}

Dans le repérage des collocations disjointes en anglais, le problème de la correction de l'étiquetage morpho-syntaxique peut également se poser, en particulier pour les couples de catégories donnant le plus souvent lieu à une ambiguïté morphologique: nom singulier / verbe à l'infinitif (result), nom pluriel / verbe à la troisième personne du singulier (results), verbe au prétérit / participe passé (treated), participe passé / adjectif ( mixed). Ainsi, une phrase comme Results of trials of selective gut decontamination have been mixed est incorrectement étiquetée par tous les programmes auxquels nous l'avons soumise. Nous reproduisons à titre d'exemple l'étiquetage attribué par le programme CLAWS $^{5}$ (utilisé pour l'étiquetage du British National Corpus) et la traduction de cette phrase par le logiciel Systran Classic 3.0.6(voir tableau 1).

Tableau 1. Étiquetage grammatical d'une phrase par le programme CLAWS et traduction de cette phrase en français par le logiciel Systran Classic

Results_NN2 of_IO trials_NN2 of_IO selective_JJ gut_NN1 decontamination_NN1 have_VHO been_VBN mixed_VVN ._.

Des résultats des épreuves de la décontamination sélective d'intestin ont été mélangés. ${ }^{7}$

La collocation non identifiée est ici mixed results (résultats mitigés), la catégorie grammaticale incorrectement attribuée à mixed par le programme d'étiquetage (et sans doute par l'analyseur de Systran) étant celle du participe passé au lieu de l'adjectif. Notons au passage que les choix erronés du logiciel d'aide à la traduction en matière de lexique (les traductions correctes sont: trials $\rightarrow$ essais, selective gut decontamination $\rightarrow$ décontamination digestive sélective) peuvent être rectifiés grâce au renseignement par l'utilisateur du dictionnaire personnalisé inclus dans le programme . Il existe même une ambiguïté syntaxique correctement traitée par le logiciel (selective modifie bien decontamination et non pas gut). De fait, les améliorations pouvant être apportées à la traduction proposée concernent essentiellement l'emploi des déterminants.

7 Le repérage des collocations disjointes pose un double problème. D'une part, leur prise en compte dans les modules automatisés décrits plus haut en ralentit considérablement le fonctionnement, en raison du supplément de mémoire qu'elle exige. D'autre part, leur repérage dans les textes à traduire nécessiterait la détermination d'un écart maximum (c'est la fonction du filtre SPAN dans le logiciel $\mathrm{TACT}^{8}$ ) et produirait le même type de ralentissement. Dans l'exemple cité plus haut, 8 mots séparent results de mixed. Une optimisation de cette fonction d'empan nécessiterait la prise en compte de statistiques de présence à l'état disjoint (on présume, par exemple, que ces statistiques révéleraient que la collocation "aspects observés» sera plus souvent disjointe que la combinaison " atteinte vasculaire ») afin de n'utiliser ces fonctions de recherche qu'en cas de nécessité. Une autre piste, moins coûteuse en mémoire, consiste à isoler des groupes de mots (parfois appelés chunks dans la littérature anglo-saxonne) dont la traduction dans une 
langue est homogène dans les corpus bilingues alignés9. Dans le cas de la suite have been mixed dans la littérature médicale, cette approche donnerait le résultat escompté puisque cette suite ne se présente qu'avec des arguments tels que results ou reviews, qui donnent lieu à la seule traduction "ont été mitigés» en français ${ }^{10}$. Cette possibilité de désambiguïsation par séquence de mots est facilitée par la distinction opérée par le système aspectuo-temporel de l'anglais entre les deux formes have been mixed et were mixed, la première étant toujours utilisée dans un contexte évaluatif, alors que la deuxième, pour la totalité des occurrences rencontrées dans IM93 ${ }^{11}$, est une vraie forme passive faisant référence à un processus s'inscrivant dans une séquence expérimentale telle qu'elle peut être décrite dans la partie Methods d'un article de recherche.

\section{L'ambiguiité syntaxique}

\subsection{Adjectifs et participes passés}

8 La résolution de l'ambiguïté syntaxique provoquée par le double statut grammatical de mixed est donc le principal écueil rencontré par le logiciel. Diverses solutions sont envisageables pour tenter de résoudre un tel problème, si tant est qu'il soit d'abord identifié : le recensement de tous les participes passés pouvant être employés comme adjectif est bien sûr nécessaire dans un premier stade, afin que l'analyse par défaut d'une forme de participe passé suivant been comme faisant partie d'une structure passive soit éventuellement suivie d'une tentative de désambiguïsation. On peut envisager trois modes de désambiguïsation dont le coût en temps de traitement est variable. Le stockage des collocations de patron syntaxique <Adjectif - Nom> constitue la solution la plus simple. L'élimination de l'interprétation active de la prédication < somebody - mix results> pourrait également être confirmée par la présence d'une combinaison telle que combine results $^{12}$ dans une base de donnée collocationnelle regroupant les verbes et leurs principaux arguments, mais on peut également imaginer la recherche en corpus (plus coûteuse en temps de traitement) d'éventuelles cooccurrences entre results et les synonymes de mix fournis par une base lexicale comme Wordnet (Fellbaum 1998). Enfin, le repérage de la collocation sous sa forme disjointe telle qu'elle se présente dans le contexte semble difficile à réaliser. En effet, les programmes de collecte automatique de collocations fonctionnent soit par repérage de mots immédiatement consécutifs (c'est le cas du générateur de collocations du logiciel TACT), soit en fournissant une série de contextes de co-occurrence de deux mots donnés dans des limites de proximité prédéfinies. Plus le nombre de mots séparant les deux termes de la collocation est élevé, plus son repérage devient aléatoire, car l'indice de probabilité de cooccurrence diminue en fonction de la distance exprimée en nombre de $\operatorname{mots}^{13}$.

\subsection{Structures coordonnées}

9 Si le problème du rattachement des groupes prépositionnels et celui des énoncés susceptibles d'induire en erreur les programmes d'analyse automatique ont donné lieu à de nombreux travaux, le problème de l'ambiguïté syntaxique générée par les structures coordonnées en langue de spécialité semble susciter un moindre intérêt, sans doute parce que la résolution de ce type d'ambiguïté nécessite un fort apport d'information lexicale dépendant du sous-langage de spécialité et difficilement généralisable à d'autres 
domaines. Parmi les structures coordonnées de l'anglais, celle du type <ADJ N1 AND N2> est d'usage fréquent, et provoque une ambiguïté dans la mesure ou l'adjectif modifie soit les deux noms coordonnés soit uniquement le premier. Un bref exemple suffira à illustrer la multiplicité d'interprétations que génère la combinaison de la prémodification nominale et de la coordination en anglais de spécialité :

(1) The ability of PET to detect cancer is based on the altered substrate requirements of malignant cells, which result from increased nucleic acid and protein synthesis and glycolysis.

$10 \mathrm{Au}$ décodage, le traducteur de cet énoncé est amené à se poser plusieurs questions concernant la structure syntaxique de la dernière partie de la phrase, increased nucleic acid and protein synthesis and glycolysis. Certaines de ces questions trouveront chez le traducteur humain une réponse immédiate grâce à l'examen du contexte et des relations sémantiques qui lient les mots entre eux, mais poseront problème à l'analyseur syntaxique automatique :

- nucleic modifie-t-il acid, l'ensemble acid and protein ou bien synthesis?

- protein est-il un prémodificateur du seul nom synthesis ou à la fois de synthesis et de glycolysis ?

- increased modifie-t-il acid, synthesis ou bien l'ensemble synthesis and glycolysis?

11 Les sources possibles d'erreurs se conjuguant, les chances d'arriver au découpage correct sans l'apport de connaissances lexicales sont réduites. Si l'on symbolise la portée des prémodifications à l'aide de crochets, le découpage correct est le suivant : increased [ [ [ [ nucleic acid] and [protein] ] synthesis] and glycolysis], et ce segment peut donc se traduire par "l'augmentation de la glycolyse et de la synthèse des protéines et de l'acide nucléique ». Les mécanismes de désambiguïsation du traducteur humain dépendent partiellement de sa connaissance de la réalité extralinguistique mais aussi d'une connaissance lexicale transmissible à la machine sous forme d'une base de données contenant à la fois les termes et les collocations de la langue de spécialité. Ainsi, si l'analyseur a accès à une telle base, les découpages supposant l'existence de nucleic protein ou de nucleic synthesis seront invalidés puisque ces séquences n'y figureront pas, et nucleic acid synthesis, inversement, sera validé. Quant à la résolution de l'ambiguïté concernant la portée de la prémodification par increased, elle repose fortement sur la connaissance extralinguistique du domaine de spécialité ${ }^{14}$, même si la fréquence des structures coordonnées à la suite de participes passés comme decreased et increased peut donner lieu à une analyse statistique de la probabilité de distributivité de la prémodification.

\section{L'acquisition automatique de collocations}

12 Les exemples cités plus haut montrent les services que peut rendre une base de données recensant les phénomènes de cooccurrence en langue de spécialité. Le repérage de combinaisons lexicales correspondant à un patron syntaxique donné est particulièrement adapté à l'outil informatique, puisque celui-ci est d'une grande puissance pour la génération de collocations tirées de textes numérisés ${ }^{15}$. Le tableau 2 nous donne un exemple des combinaisons de deux mots consécutifs obtenues à partir d'un corpus de 200000 mots composé d'articles portant sur la gastro-entérologie. On remarque une grande homogénéité, typique de la langue médicale (et peut-être de la langue scientifique en général) quant aux catégories grammaticales représentées, puisque 18 collocations sur 
20 sont du type <Nom - Adjectif>. Par ailleurs, l'examen de ces dernières révèle un clivage assez net entre les termes (formes relevant du lexique spécialisé), et les collocations proprement dites, dont les bases (aspect, augmentation) n'appartiennent pas au vocabulaire spécialisé. Le problème de l'automatisation du tri entre termes et collocations est l'une des difficultés rencontrées par les programmes d'extraction terminologique, pour lesquels des suites comme aspects observés ou augmentation localisée font partie du «bruit » à éliminer. Nous allons tenter de décrire un modèle inverse, visant à opérer l'extraction des seules collocations.

Tableau 2. Combinaisons lexicales de fréquence au moins égale à 4 commençant par la lettre $A$ (corpus de gastro-entérologie)

\begin{tabular}{|c|c|c|}
\hline FREQ & WORD1 & WORD2 \\
\hline 12 & atteintes & inflammatoires \\
\hline 8 & anses & grêles \\
\hline 7 & aspects & radiologiques \\
\hline 7 & atrophie & villositaire \\
\hline 6 & abcès & hépatiques \\
\hline 6 & anses & intestinales \\
\hline 6 & aspects & observés \\
\hline 6 & atteintes & vasculaires \\
\hline 5 & adénomes & hépatocellulaires \\
\hline 5 & aspect & pseudo-tumoral \\
\hline 4 & abdominal & aigu \\
\hline 4 & anastomose & gastro-jéjunale \\
\hline 4 & anatomie & pathologique \\
\hline 4 & angiomes & géants \\
\hline 4 & antérieur & gauche \\
\hline 4 & arcades & dentaires \\
\hline 4 & aspect & nodulaire \\
\hline 4 & aspect & radiologique \\
\hline 4 & augmentation & localisée \\
\hline
\end{tabular}




\begin{tabular}{|l|l|l|}
\hline 4 & axes & vasculaires \\
\hline
\end{tabular}

13 Clas suggère un classement des collocations lexicales en divers groupes basés sur une fonction syntagmatique qui intègre les six catégories suivantes (1994: 578):

1. verbe et nom, où le verbe a un contenu sémantique très général proche simplement de « faire » (prononcer un discours);

2. nom et adjectif (rude épreuve, marque distinctive) ;

3. adverbe et adjectif (vachement bon);

4. verbe et adverbe (boire goulûment)

5. nom (sujet) et verbe (la cloche sonne, le chat miaule, l'abeille bourdonne) ;

6. marquage de la quantité (unité ou collectif) du nom (essaim d'abeilles, troupeau de vaches, pincée de sel, barre de chocolat).

Les exemples donnés ici correspondent à des degrés de figement et de lexicalisation divers, et les restrictions s'appliquant à certaines des catégories définies par Clas pourraient être élargies ${ }^{16}$. Par ailleurs, les associations décrites dans le cadre des quatre premières catégories semblent constituer un choix de combinatoire lexicale possible parmi d'autres, alors que celles des catégories 5 et 6 ne peuvent s'accommoder de variations (le marquage de la quantité fait d'ailleurs l'objet d'explications et d'exercices dans la plupart des grammaires de l'anglais). La Figure 1 représente un schéma combinatoire des différentes catégories morpho-syntaxiques établi à partir des catégories décrites plus haut.

Figure 1. Combinatoire collocationnelle des catégories morpho-syntaxiques

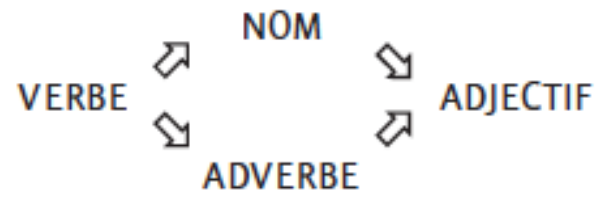

\section{Outils informatiques utilisés et extraction des combinaisons <Verbe - Nom>}

Nous avons utilisé pour tester notre technique d'extraction un corpus constitué de 58 articles du Journal of the American Medical Association. La longueur totale du corpus est de 134000 mots. Le calcul de l'indice de probabilité de cooccurrence des collocants a été effectué à l'aide du logiciel TACT. Nous avons testé deux méthodes d'extraction correspondant à deux accès possibles aux collocations <Adjectif - Nom> en anglais selon la Figure 1. L'une utilise comme point de départ les cooccurrents des verbes (l'ordre suivi est donc Verbe $\rightarrow$ Nom $\rightarrow$ Adjectif) et l'autre utilise comme point de départ les cooccurrents des adverbes (l'ordre suivi est donc Adverbe $\rightarrow$ Adjectif $\rightarrow$ Nom). 


\subsection{Extraction à partir des verbes}

16 Notre hypothèse de départ est la suivante: les termes, constitués essentiellement de groupes nominaux fréquemment concentrés en groupes compacts dans la prose spécialisée entrent moins fréquemment en cooccurrence avec les verbes que les noms qui sont la base des collocations de la langue de spécialité. En commençant donc par extraire les combinaisons formées des verbes et de leurs sujets ou objets typiques, puis en extrayant les combinaisons de ces noms avec les adjectifs, on obtiendra une plus forte proportion de collocations par rapport aux termes qu'en extrayant directement les combinaisons de la forme Adjectif - Nom.

Tableau 3 : Combinaisons lexicales $<$ Verbe - Nom $>$ du corpus de cardiologie de z-score ${ }^{17}$ supérieur à 13

\begin{tabular}{|c|c|c|}
\hline Verbe & Collocant & z-score \\
\hline impair & tolerance & 31,809 \\
\hline affect & hemispheres & 28,936 \\
\hline take & medications & 25 \\
\hline undergo & arteriography & 24,883 \\
\hline impair & glucose & 23,417 \\
\hline perform & anesthesia & 22,939 \\
\hline prevent & reaccumulation & 21,952 \\
\hline affect & lesion & 20,412 \\
\hline elevate & fibrinogen & 19,916 \\
\hline decrease & libido & 19,817 \\
\hline influence & environment & 19,453 \\
\hline assess & surveys & 16,683 \\
\hline provide & overview & 16,379 \\
\hline provide & discrimination & 16,379 \\
\hline treat & atenolol & 16,195 \\
\hline influence & genetics & 15,842 \\
\hline make & decisions & 15,488 \\
\hline
\end{tabular}




\begin{tabular}{|l|l|l|}
\hline base & scoring & 15,375 \\
\hline establish & certainty & 15,156 \\
\hline appear & pericardiectomy & 15,156 \\
\hline identify & search & 15,092 \\
\hline elevate & levels & 14,622 \\
\hline implant & system & 14,139 \\
\hline \hline reduce & costs & 14,019 \\
\hline involve & extractions & 14,012 \\
\hline recommend & chemoprophylaxis & 13,761 \\
\hline cause & bacteremia & 13,48 \\
\hline impair & consciousness & 13,238 \\
\hline require & injection & 13,13 \\
\hline require & hospitalization & 13,13 \\
\hline
\end{tabular}

17 Après avoir évalué divers programmes d'étiquetage automatique, nous avons soumis notre corpus à un étiquetage morpho-syntaxique selon les normes du corpus LOB (Lancaster-Oslo/Bergen) ${ }^{18}$. Toutes les combinaisons de type <Verbe - Nom> contenant des verbes de fréquence supérieure ou égale à 10 ont ensuite été isolées et recopiées dans une base de données afin d'effectuer des mesures statistiques et des regroupements par indexation automatique. Le Tableau 3 reproduit les 30 combinaisons d'indice de probabilité de cooccurrence ( $z$-score) le plus élevé, exception faite des hapax ${ }^{19}$.

18 La majorité des combinaisons relevées correspondent au patron <verbe + complément d'objet>, mais faute d'un étiquetage syntaxique détaillé (full parsing), certaines ne correspondent pas à cette structure syntaxique: on trouve également des noms appartenant à des groupes prépositionnels (treat with atenolol, establish with certainty, identify in / with / through a search) et d'autres qui sont en fait des prémodificateurs d'autres noms à l'intérieur de termes complexes (impair glucose correspond à des emplois de la suite impaired glucose tolerance, elevate fibrinogen à elevated fibrinogen levels) ${ }^{20}$.

19 Le processus a ensuite été répété en partant de la liste des noms qui entraient en cooccurrence avec les verbes déjà isolés. Comme les cooccurrents recherchés étaient essentiellement des adjectifs, nous avons cependant diminué la taille de la fenêtre utilisée. Alors que celle-ci était de cinq mots à gauche et à droite des formes verbales repérées, afin de prendre en compte les nombreuses formes passives employées dans la prose médicale, nous n'avons recherché les cooccurrents que parmi les mots qui précédaient immédiatement le nom ${ }^{21}$. Le Tableau 4 donne la liste des combinaisons de patron syntaxique <Adjectif - Nom> ainsi obtenues. Le classement par z-score descendant 
fait apparaître que cet indice de probabilité de cooccurrence favorise les termes qui sont de fréquence d'occurrence supérieure (la fréquence d'occurrence des combinaisons dans le corpus est donnée en colonne 3). De fait, la quasi-totalité des vingt premières combinaisons du tableau sont des termes de la langue médicale. On voit cependant cette tendance s'inverser dans la deuxième moitié du tableau à partir des combinaisons dont le z-score est inférieur à 20, la plupart de celles-ci n'étant pas des termes mais des collocations. La méthode suggérée initialement pour isoler les collocations des termes ne semble donc pouvoir fonctionner que si l'on élimine les combinaisons dont le z-score est élevé. Cette technique a toutefois ses limites, car certains termes complexes (coronary disease, pericardial disease) ont un z-score peu élevé en raison de la grande fréquence d'emploi dans le corpus de l'un de leurs composants (ici le mot disease).

Tableau 4 : Combinaisons lexicales <Adjectif - Nom > dont le nom entre en co-occurrence avec les verbes du corpus médical

\begin{tabular}{|l|l|l|}
\hline Combinaison <ADJ - N> & Freq. & zscore \\
\hline myocardial infarction & 36 & 149,197 \\
\hline coronary artery & 45 & 103,363 \\
\hline diastolic dysfunction & 36 & 99,75 \\
\hline visceral fat & 7 & 97,619 \\
\hline sclerosing agents & 3 & 69,624 \\
\hline blocking agents & 3 & 69,624 \\
\hline sexual dysfunction & 30 & 68,467 \\
\hline venous hypertension & 8 & 60,397 \\
\hline systolic dysfunction & 21 & 60,097 \\
\hline ischemic stroke & 14 & 59,808 \\
\hline evolving conditions & 2 & 59,171 \\
\hline inotropic agents & 2 & 56,847 \\
\hline abdominal fat & 2 & 55,783 \\
\hline annual mortality & 4 & 54,62 \\
\hline false-negative rate & 7 & 54,531 \\
\hline temporal artery & 52,409 \\
\hline \hline
\end{tabular}




\begin{tabular}{|c|c|c|}
\hline environmental factors & 4 & 52,231 \\
\hline transient bacteremia & 2 & 45,82 \\
\hline intra-abdominal fat & 2 & 45,533 \\
\hline post-myocardial infarction & 2 & 42,26 \\
\hline progressive increase & 2 & 40,566 \\
\hline high-risk conditions & 3 & 39,644 \\
\hline basilar artery & 2 & 37,083 \\
\hline true-positive rate & 2 & 34,86 \\
\hline true-negative rate & 2 & 34,86 \\
\hline sedimentation rate & 2 & 34,86 \\
\hline false-positive rate & 3 & 33,036 \\
\hline cardiovascular disease & 6 & 31,748 \\
\hline perioperative risk & 2 & 31,032 \\
\hline cardiac conditions & 4 & 30,977 \\
\hline antihypertensive therapy & 6 & 30,43 \\
\hline hypertensive patients & 24 & 29,578 \\
\hline antihypertensive agents & 3 & 329,168 \\
\hline proven stroke & 2 & 28,371 \\
\hline lacunar stroke & 2 & 28,371 \\
\hline minor stroke & 3 & 26,871 \\
\hline blind study & 2 & 25,459 \\
\hline emotional symptoms & 2 & 24,655 \\
\hline atherogenic type & 3 & 23,829 \\
\hline subcutaneous fat & 2 & 23,72 \\
\hline embolic stroke & 2 & 23,136 \\
\hline moderate hypertension & 3 & 22,566 \\
\hline
\end{tabular}




\begin{tabular}{|c|c|c|}
\hline congestive symptoms & 2 & 22,492 \\
\hline underlying risk & 2 & 21,898 \\
\hline early changes & 2 & 21,53 \\
\hline cerebrovascular disease & 2 & 20,284 \\
\hline diuretic therapy & 3 & 19,814 \\
\hline medical conditions & 2 & 19,637 \\
\hline vascular disease & 3 & 18,563 \\
\hline genetic factors & 3 & 18 \\
\hline increased risk & 4 & 17,995 \\
\hline selected patients & 4 & 17,662 \\
\hline at-risk patients & 6 & 17,551 \\
\hline surgical mortality & 2 & 17,54 \\
\hline human study & 3 & 16,958 \\
\hline asymptomatic patients & 8 & 16,391 \\
\hline increased insulin & 2 & 16,226 \\
\hline coronary risk & 7 & 16,135 \\
\hline treated patients & 3 & 15,295 \\
\hline diuretic-treated patients & 3 & 15,295 \\
\hline medical therapy & 3 & 14,68 \\
\hline general use & 2 & 14,048 \\
\hline premature study & 2 & 13,846 \\
\hline male patients & 5 & 13,787 \\
\hline high-risk patients & 5 & 13,787 \\
\hline small size & 2 & 13,351 \\
\hline large artery & 3 & 13,293 \\
\hline captopril-treated patients & 3 & 13,19 \\
\hline
\end{tabular}




\begin{tabular}{|l|l|l|}
\hline recent study & 3 & 13,047 \\
\hline higher risk & 3 & 12,545 \\
\hline conventional therapy & 2 & 12,533 \\
\hline \hline coronary disease & 8 & 11,757 \\
\hline \hline higher rate & 3 & 11,461 \\
\hline additional therapy & 2 & 11,071 \\
\hline \hline greater risk & 2 & 10,589 \\
\hline physical symptoms & 2 & 9,424 \\
\hline high mortality & 2 & 9,344 \\
\hline antibiotic therapy & 3 & 9,32 \\
\hline \hline specifi c therapy & 2 & 8,968 \\
\hline symptomatic patients & 5 & 8,581 \\
\hline placebo-treated patients & 2 & 7,028 \\
\hline individual patients & 3 & 6,752 \\
\hline sexual symptoms & 2 & 6,519 \\
\hline low-risk patients & 2 & 6,464 \\
\hline consecutive patients & 6,464 \\
\hline female patients & 2,007 \\
\hline pericardial disease & 253 \\
\hline clinical symptoms & 2 & \\
\hline
\end{tabular}

\subsection{Extraction à partir des adverbes}

20 La deuxième technique utilisée pour l'isolation des collocations des termes complexes repose sur une caractéristique connue des adjectifs relationnels, qui rentrent dans la composition des termes de patron syntaxique <Adjectif - Nom>. Daille (2001) fait remarquer qu'outre les critères de repérage purement morphologiques, telle la présence majoritaire de certains suffixes de dérivation, on peut également utiliser certaines caractéristiques syntaxiques ou sémantiques des adjectifs relationnels, par exemple le fait qu'ils ne sont pas combinables avec certains adverbes, en particulier les adverbes de degré (on ne dit pas *une production très laitière). Nous avons donc tenté de repérer 
automatiquement uniquement les adjectifs modifiés par des adverbes dans notre corpus, puis d'extraire les noms entrant en cooccurrence avec ces adjectifs, cette méthode ayant pour but d'éliminer les combinaisons contenant des adjectifs relationnels tels que coronary ou pericardial, qui constituent le plus souvent des termes de la langue médicale.

21 Nous avons donc isolé les 1057 combinaisons <Adverbe - Adjectif> de notre corpus médical. Après élimination manuelle des suites contenant certains adjectifs relationnels ( atherogenic, bactericidal, hyperinsulinemic, metastatic, occlusive, oral, vascular), de celles dont les adverbes ne modifiaient pas l'adjectif qu'ils précédaient (therefore, still, thus, likewise, perhaps, also, once, enough, prior), et de celles contenant des adverbes pouvant précéder des adjectifs relationnels ${ }^{22}$, il restait 869 occurrences d'un adjectif précédé d'un adverbe ${ }^{23}$. Ces occurrences regroupaient 596 combinaisons distinctes, contenant 342 adjectifs distincts. Nous avons ensuite extrait du corpus 3121 occurrences du patron syntaxique <Adjectif Nom> qui contenaient ces 342 adjectifs et formaient 2034 combinaisons distinctes.

Le Tableau 5 donne la liste des 67 séquences de fréquence supérieure à 4 dans notre corpus. La précision de cette méthode d'extraction des collocations est nettement supérieure à la précédente, la liste contenant très peu de termes complets ${ }^{24}$ (on relève cependant un certain nombre d'emplois de l'adjectif low précédant le premier terme d'une lexie complexe comme ejection fraction ou compliance rate). Quant à son rappel, il est difficile à évaluer sans une exploitation manuelle intégrale du corpus. Un certain nombre des collocations du Tableau 4 ne sont pas repérées ici, soit parce que les adjectifs ne sont pas modifiés par des adverbes dans notre corpus (emotional symptoms, general use) soit parce que leur caractéristiques sémantiques n'autorisent pas une prémodification adverbiale (underlying risk). La technique utilisée fait que le taux de rappel doit logiquement augmenter avec la taille du corpus, puisqu'il suffit d'une occurrence postadverbiale d'un adjectif pour que celui-ci soit ajouté à la liste des éventuels co-occurrents des noms qui servent de base à la deuxième passe.

Tableau 5. Combinaisons lexicales <Adjectif - Nom> dont l'adjectif entre en co-occurrence avec les adverbes du corpus médical

\begin{tabular}{|l|l|l|}
\hline \multicolumn{1}{|c|}{ Adjectif } & \multicolumn{1}{|c|}{ Nom } & Freq. \\
\hline physical & examination & 50 \\
\hline increased & risk & 23 \\
\hline clinical & trials & 22 \\
\hline clinical & probability & 19 \\
\hline predictive & value & 18 \\
\hline diagnostic & test & 17 \\
\hline relative & risk & 15 \\
\hline clinical & findings & 15 \\
\hline recent & studies & 11 \\
\hline
\end{tabular}




\begin{tabular}{|c|c|c|}
\hline high & risk & 11 \\
\hline clinical & assessment & 11 \\
\hline toxic & effects & 10 \\
\hline physical & findings & 10 \\
\hline malignant & effusions & 10 \\
\hline increased & prevalence & 10 \\
\hline high & levels & 10 \\
\hline diagnostic & tests & 10 \\
\hline higher & levels & 9 \\
\hline adverse & effects & 9 \\
\hline sensitive & thromboplastins & 8 \\
\hline lower & liver & 8 \\
\hline lower & levels & 8 \\
\hline higher & risk & 8 \\
\hline early & detection & 8 \\
\hline controlled & trials & 8 \\
\hline small & number & 7 \\
\hline physical & diagnosis & 7 \\
\hline lower & edge & 7 \\
\hline low & probability & 7 \\
\hline clinical & presentation & 7 \\
\hline antimicrobial & therapy & 7 \\
\hline total & cholesterol & 6 \\
\hline systemic & signs 6 & 6 \\
\hline sensitive & thromboplastin & 6 \\
\hline recent & report & 6 \\
\hline
\end{tabular}




\begin{tabular}{|c|c|c|}
\hline negative & test & 6 \\
\hline malnourished & patients & 6 \\
\hline malignant & effusion & 6 \\
\hline lower & extremities & 6 \\
\hline increased & levels & 6 \\
\hline greater & risk & 6 \\
\hline functional & status & 6 \\
\hline diagnostic & testing & 6 \\
\hline clinical 6 & management & 6 \\
\hline clinical & examination & 6 \\
\hline clinical & evaluation & 6 \\
\hline active & gastritis & 6 \\
\hline systemic & chemotherapy & 5 \\
\hline substantial & proportion & 5 \\
\hline significant & number & 5 \\
\hline responsive & thromboplastins & 5 \\
\hline recent 5 & study & 5 \\
\hline recent & reports & 5 \\
\hline protective & threshold & 5 \\
\hline positive & family & 5 \\
\hline normal & ejection & 5 \\
\hline new & new lot & 5 \\
\hline new & cases & 5 \\
\hline low & ejection & 5 \\
\hline low & compliance & 5 \\
\hline important & role & | 5 \\
\hline
\end{tabular}




\begin{tabular}{|l|l|l|}
\hline effective & treatment & 5 \\
\hline different & thromboplastins & 5 \\
\hline diagnostic & criteria & 5 \\
\hline diagnostic & accuracy & 5 \\
\hline clinical & practice & 5 \\
\hline asymptomatic & patients & 5 \\
\hline
\end{tabular}

\section{Conclusion}

L'exploitation d'un corpus étiqueté morpho-syntaxiquement permet des regroupements des phénomènes de cooccurrence par patrons syntaxiques qui peuvent s'avérer utiles dans la démarche consistant à séparer les candidats termes des " candidats collocations ", en particulier dans le cas des structures faisant intervenir la prémodification adjectivale du nom. La deuxième méthode que nous avons testée obtient une précision correcte, mais demande à être testée sur un corpus de taille supérieure. L'amélioration de son rappel doit être envisagée à partir d'une liste de cooccurrents correspondant au patron syntaxique <Adjectif - Nom> n'ayant pas fait l'objet d'une sélection préalable. La distinction entre termes et collocations de la langue spécialisée reste parfois difficile à opérer, et nécessite à un stade ultérieur la prise en compte de traits sémantiques réglementant la combinatoire des noms et des adjectifs. Dans cette optique, l'accès à la liste des candidats termes rejetés lors du processus de la validation terminologique opérée par les linguistes et les spécialistes du domaine lors d'études antérieures pourrait s’avérer précieux.

\section{BIBLIOGRAPHIE}

Atwell, Eric. et al. 2000. « A comparative evaluation of modern English corpus grammatical annotation schemes ». ICAME Journal 24, 7-23.

Bourigault, Didier et Christian Jacquemin. 2000. « Construction de ressources terminologiques ». In Pierrel, Jacques (dir.), Ingénierie des langues. Paris : Hermès, chapitre 9.

Church, K. et P. Hanks. 1989. « Word association norms, mutual information and lexicography ». Proceedings of the 27th Conference of the ACL, 76-83. Amsterdam : John Benjamins.

Fellbaum, Christiane (dir.). 1998. WordNet: An Electronic Lexical Database. Cambridge, MA : MIT Press. 
Gaussier, Eric. 2001. « General considerations on bilingual terminology extraction ». In Bourigault, Didier, Christian Jacquemin et Marie-Claude L'Homme (dir.), Recent advances in computational terminology. Amsterdam : John Benjamins, 167-184.

Humbley, John, Christine Chodkiewicz et Didier Bourigault. 1999. « Some techniques and tools for tailor-made terminologies ». In Sandrini, P. (dir.), Terminology and Knowledge Engineering, TKE '99. Vienne : TermNet, 378-390.

Langlois, Lucie et Pierre Plamondon. 1998. «Le répérage automatique de collocations équivalentes à partir de bitextes ». In Fontenelle, Thierry et al (dir.), Euralex'98: Proceedings of the Eighth Euralex International Congres. Liège : Université de Liège, 175-186.

L'Homme, Marie-Claude. 1998. « Définition du statut du verbe en langue de spécialité et sa description lexicographique ». Cahiers de lexicologie 73/2, 61-84.

Maniez, François. 2001. «The use of electronic corpora and lexical frequency data in solving translation problems ». In Altenberg, Bengt \& Sylviane Granger (dir.), Lexis in Contrast. Amsterdam : John Benjamins.

Maniez, François. 2001. « Désambiguïsation syntaxique des groupes nominaux en langue spécialisée : le cas des adjectifs en anglais » Actes du colloque TALN, Tours, 2-5 juillet 2001, Tome 1, 273-282.

Mel'cuk, Igor. 1984. Dictionnaire explicatif et combinatoire du français contemporain. Montréal : Les Presses de l'Université de Montréal.

Williams, Geoffrey. 1998. « Collocational networks: Interlocking patterns of lexis in a corpus of plant biology research articles ». International Journal of Corpus Linguistics 3/1, 151-171.

\section{ANNEXES}

\section{Formule de calcul du z-score}

La formule de calcul du z-score est la suivante :

$\mathrm{Z}=($ fréquence $\mathrm{du}$ collocant-E)/Écart type

Écart type $=$ Racine carrée de [Longueur du mini-texte $* \mathrm{P} *(1-\mathrm{P})$ ]

$\mathrm{E}=\mathrm{P} *$ longueur du mini-texte, et :

$\mathrm{P}=$ Fréquence du collocant sur l'ensemble du texte / longueur du texte.

Le mini-texte représente le nombre total de mots à gauche et à droite du mot-cible (10 par défaut) constituant la « fenêtre » à l'intérieur de laquelle les collocants sont recherchés. La prise en compte d'une fenêtre de plus faible taille augmente donc la valeur du z-score.

Une autre mesure fréquemment employée est le mutual information (MI) score. Le mode de calcul du MI score est donné par la formule suivante, dans laquelle $\mathrm{P}(\mathrm{X}, \mathrm{Y})$ est la probabilité que le mot $\mathrm{X}$ entre en cooccurrence avec le mot $\mathrm{Y}, \mathrm{P}(\mathrm{X})$ et $\mathrm{P}(\mathrm{Y})$ représentant la fréquence de ces deux mots dans le corpus :

$$
M I(X, Y)=\log 2 \quad \frac{P(X, Y)}{P(X) * P(Y)}
$$

Pour une description détaillée de l'utilisation du MI score, voir Church \& Hanks (1989). 


\section{NOTES}

1. Bourigault et Jacquemin (2000) soulignent la relative facilité de cette extraction dans une langue comme l'anglais, qui construit ses termes complexes essentiellement par juxtaposition d'unités lexicales pleines, alors que le français use abondamment des prépositions et des déterminants, phénomène qui rend la distinction entre terme et syntagme libre plus difficile à saisir par les outils automatiques.

2. Traitement Automatique des Langues Naturelles.

3. Les corpus arborés de grande taille étant rares en langue de spécialité, nous avons toutefois dû nous contenter d'utiliser des corpus étiquetés morpho-syntaxiquement dans le cadre de cette étude.

4. Ce corpus, compilé à partir des sites des Universités de Grenoble 2, Lyon 1 et Rennes 2, totalise deux millions de mots.

5. Ce programme a été mis au point par le Unit for Computer Research on the English Language (UCREL) de l'Université de Lancaster.

6. SYSTRAN ${ }^{\circledR}$ Classic. Copyright @ $1968-1999$ SYSTRAN. <info@systransoft.com>

7. Une traduction possible de l'original serait: «Les essais portant sur la décontamination digestive sélective ont connu des résultats mitigés».

8. TACT (Copyright (C) 1989 John Bradley, University of Toronto), logiciel accessible à l'URL suivante : <http://www.chass.utoronto.ca: 8080/cch/tact.html>

9. Alors que l'extraction terminologique bilingue progresse à grandes enjambées, cf. notamment Gaussier (2001), le repérage automatique des collocations dans les corpus bilingues alignés demeure un exercice beaucoup plus délicat comme le montrent Langlois et Plamondon (1998).

10. Ceci est en fait dû à certaines restrictions syntaxiques qui apparaissent dans l'utilisation des collocants de mixed: les noms pour lesquels la traduction de mixed par l'adjectif "mitigé " (comme emotions ou feelings) n'est pas recommandée par les dictionnaires sont ceux auxquels mixed est associé en tant qu'adjectif épithète (le modèle le plus fréquent étant have mixed emotions / feelings), alors que cette traduction semble possible pour tous les noms employés dans une structure attributive, dont le sémantisme semble par ailleurs davantage lié à la notion d'évaluation (results, reviews, reactions, rating, marks, profile).

11. Le corpus IM93 regroupe la totalité des articles parus en 1993 dans quatre revues médicales de langue anglaise et totalise environ 12 millions de mots.

12. L'examen du corpus IM93 nous montre que la collocation combine results est présente sous sa forme liée dans 46 articles (le verbe s'y présente sous les trois formes combine, combined et combining), alors que tous les emplois liés de mix et de results se présentent sous la forme mixed results, mixed étant un adjectif.

13. Cette distance entre les deux membres d'une collocation constitue également un obstacle à la compréhension des humains. Lors d'une épreuve écrite donnée à des étudiants de DESS, 50 \% d'entre eux ont interprété have been mixed comme étant une forme passive, mais pensaient avoir déjà rencontré la collocation mixed results, et prétendaient qu'une forme plus brève comme Results have been mixed ne leur aurait pas posé de problème d'interprétation.

14. Même si la perception d'une relation proche de l'hyperonymie entre alter et increase joue sans doute un rôle.

15. Il faut toutefois signaler l'importance quantitative du « bruit » par rapport au signal si l'on se borne à repérer les séquences de mots consécutifs sans avoir recours à aucun type d'étiquetage préalable. Lors de tests portant sur les collocations du français en langue médicale, l'élimination des mots-outils fait chuter le nombre de collocations conservées pour stockage à $6 \%$ des formes initialement sélectionnées par le logiciel TACT. La longueur des mots n'étant que l'un des critères 
retenus pour l'élagage des listes des séquences générées par le logiciel, l'ajout d'un programme de traitement qui élimine certaines formes lexicales s'avère nécessaire.

16. Par exemple, faut-il nécessairement limiter les collocations de la première catégorie à celles qui comprennent un verbe support? Dans le cas des collocations anglaises set / beat (ou break) / hold a record (établir / battre / détenir un record), cela reviendrait à n'accepter que la première et pas les autres dans cette catégorie.

17. Voir annexe pour le calcul de z-score.

18. Le projet AMALGAM, décrit par Atwell et al. (2000), mettait jusqu'à une date récente à la disposition des internautes plusieurs programmes d'étiquetage grammatical de l'anglais. L'adresse est la suivante <http://www.comp.leeds.ac.uk/amalgam/amalgam/amalghome.htm>.

19. Les cooccurrences entre verbe et nom de fréquence 1 sont phraséologiquement peu significatives. Humbley et al. (1999) font cependant état de l'identification d'un nombre important de termes ne comportant qu'une seule occurrence dans un corpus bilingue aligné dans le domaine des droits de l'homme : à la suite d'une extraction manuelle des termes anglais et de leur appariement à des équivalents de traduction français, plus du tiers des termes identifiés étaient des hapax, et plus de la moitié des hapax relevés ont été identifiés comme étant des termes.

20. Ces deux derniers exemples découlent cependant d'un étiquetage morpho-syntaxique incorrect des participes passés employés comme adjectifs; il s'agit d'une erreur fréquente des programmes d'étiquetage automatique. Pour une discussion plus détaillée des erreurs d'étiquetage dans l'analyse des textes en langue de spécialité, voir Maniez (2001).

21. Ceci explique les valeurs sensiblement plus élevées du z-score dans le Tableau 4, puisque celui-ci est inversement proportionnel à la taille de cette fenêtre.

22. Certains adverbes tels que largely semblent précéder majoritairement les adjectifs relationnels, dans des contextes tels que Ocular rosacea is largely vascular in its origin ou The residual symptoms are largely cognitive / vegetative.

23. Nous avons retenu les deux classes d'adverbe étiquetées RB (correspondant aux adverbes à dérivation en -ly) et QL (qualifier, contenant les adverbes too, least, very, as, so, more et less).

24. L'élimination totale du bruit est difficile si l'on souhaite recenser toutes les collocations, car certains adjectifs ont des emplois relationnels sans qu'ils entrent pour autant exclusivement dans la composition de termes. Par exemple, la combinaison la plus fréquente de notre liste, physical examination, est un terme, mais ce n'est pas nécessairement le cas de physical findings et de physical diagnosis.

\section{RÉSUMÉS}

La grande majorité des applications de traitement automatique des langues qui ont pour domaine les langues de spécialité sont des outils d'extraction terminologique. Elles se concentrent donc naturellement sur l'identification des groupes nominaux et des groupes prépositionnels ou prémodificateurs qui leur sont associés. En nous fondant sur un corpus bilingue anglais-français composé d'articles de recherche médicale, nous proposons un modèle d'extraction phraséologique semi-automatisée, dont le point de départ est la recherche des co-occurrents les plus fréquents des formes verbales du texte original (l'anglais), étiqueté morpho-syntaxiquement (POS-tagged). L'indice de probabilité de co-occurrence (z-score) permet dans un premier temps 
d'établir la liste des collocants les plus fréquents d'un verbe donné dans les classes des adverbes et des noms. On recherche ensuite les adjectifs qui entrent en co-occurrence avec les adverbes et les noms relevés lors de la première étape. Une deuxième méthode utilisant les adverbes comme point de départ de la recherche a été testée et semble donner de meilleurs résultats.

The great majority of natural language processing applications that are meant for specialized languages are terminological extraction tools. They consequently tend to focus on methods for identifying noun phrases and prepositional phrases as well as the words that modify them. Using an English-to-French translation corpus of medical research articles, we have tried to describe a semi-automatic phraseological extraction model by focusing primarily on the retrieval of the collocates of the most frequently used verb forms in the POS-tagged original (i.e., the English) text. The co-occurrence probability index (Tact's z-score) makes it possible to list the most frequent collocates of a particular verb within the class of adverbs or nouns. Adjectives that cooccur with the adverbs and nouns that were extracted in the first phase are then examined. Another method based on the retrieval of the collocates of adverbs was tried and seems to yield better results.

INDEX

Keywords : corpus linguistics, ESP, medical English, noun phrase, phraseology, terminological extraction

Mots-clés : anglais de spécialité, anglais médical, extraction terminologique, groupe nominal, linguistique de corpus, phraséologie

\section{AUTEUR}

\section{FRANÇOIS MANIEZ}

